\title{
Optimization of the Surfboard Fin Shape using Computational Fluid Dynamics and Genetic Algorithms
}

\author{
Konstantinos Sakellariou, Zeeshan A. Rana, Karl W. Jenkins \\ School of Aerospace, Transport Systems and Manufacturing, Cranfield \\ University, College Road, Cranfield, MK43 OAL, Bedfordshire, UK
}

Corresponding Author: Zeeshan A. Rana (zeeshan.rana@cranfield.ac.uk)

Abstract: During the sport of wave surfing, the fins on a surfboard play a major role on the overall performance of the surfer. This article presents the optimization of a surfboard fin shape, using coupled Genetic Algorithms with the FLUENT ${ }^{\oplus}$ solver, aiming at the maximization of the lift per drag ratio. The design-variable vector includes six components namely the chord length, the depth and the sweep angle of the fin as well as the maximum camber, the maximum camber position and the thickness of the hydrofoil (the four-digit NACA parametrization). The Latin Hypercube Sampling technique is utilized to explore the design space, resulting in 42 different fin designs. Fin and control volume models are created (using CATIA $^{\circledR}$ V5) and meshed (unstructured using ANSYS ${ }^{\circledR}$ Workbench). Steady-state computations were performed using the FLUENT ${ }^{\circledR}$ SST $k-\omega$ (Shear Stress Transport $k-\omega)$ turbulence model at the velocity of $10 \mathrm{~m} / \mathrm{s}$ and $10^{\circ}$ angle of attack. By using the obtained lift and drag values, a Response Surface based Model (RSM) was constructed with the aim to maximize the lift-to-drag ratio. The optimization problem was solved using the genetic algorithm provided by the MATLAB $^{\circledR}$ optimization toolbox and the RSM was iteratively improved. The resultant optimal fin design is compared with the experimental data for the fin 
demonstrating an increase of lift-to-drag ratio by approximately $62 \%$ for the given angle of attack of $10^{\circ}$.

Keywords: Genetic Algorithm, Computational Fluid Dynamics (CFD), Response Surf ace based Model (RSM), Latin Hypercube sampling, Optimization, Surfboard Fin.

\section{Introduction}

The shape of a surfboard used during the sport of wave surfing has evolved through various designs over the past several years which included introduction of $\mathrm{V}$-bottoms and twin fins ${ }^{1,2}$. The surfboard fins generate lift with lateral direction opposed to the water flow, thereby stabilizing the board's trajectory and providing maneuverability through changes of weight distribution of the surfer on the surfboard. A third fin was introduced to the surfboards, when Simon Anderson won the 1981 Bells Classic, to avoid the problem of spinning out of the surf in large waves ${ }^{1,3}$. The surfboards initially came with the fins permanently attached to them. However, in mid-nineties, the idea of fin-boxes was introduced, and this concept allowed the surfers to change the fins on their surfboards accordingly. A surfboard fin is a hydrofoil installed at the rear part of the surfboard with the aim to improve the maneuverability of the surfer.

Lift and drag of the surfboard fin play an important role in improving the manoeuvrability and performance of the surfer. The lift is related to the speed of the fin and the hence the surfer. Fin designs which induce more lift, move more quickly. To effectively understand, the connection between lift and speed on surfboard fins, a small reference will be made to lift on sailboats. In the case of 
sailboats, these are sucked forward by the sail, resulting in an increase of the sailboat speed. Consequently, increased wind speed results in an increase of the produced lift. This procedure feeds itself until the boat moves faster in comparison with wind speed and towards the wind direction. In a similar way, efficient fin designs which induce higher amounts of lift move faster ${ }^{4}$.

As far as drag is concerned, it is a force parallel to the water flow with a backwards direction. Consequently, drag is a force which slows down the surfboard and decreases the ability of the fin to produce lift. It must be mentioned that surfers can surf using surfboards with draggy fins as they allow them to stick easily to the wave. However, the majority of the surfboard manufacturers are trying to maximise lift and at the same time minimise drag as this combination allows surfers to accelerate efficiently, direct the surfboard more easily and position themselves better in the wave. Finally, finding an efficient fin shape is a complicated procedure, as a fin design which performs well in straight movement may underperform when turning and vice versa ${ }^{4}$.

The increasing demand for improvements in the fin performance, due to the high competition and professionalism in surfing, has resulted in an increasing interest in investigating the hydrodynamics of a surfboard fin. Over the years, computational fluid dynamics (CFD) has played a major role in the aerodynamic performance improvements for various sectors and thus has been found to be a very suitable tool to study the hydrodynamic performance of the surfboard fins. A virtual prototype of the fin can be analysed eliminating the need to conduct experiments in cavitation/wind tunnels, consequently resulting in huge time and cost savings. 
The radical increase in the computational power over the last few decades has created new prospects for CFD applications. In specific, CFD coupled with optimisation algorithms has been found to be beneficial in supporting product design and development. CFD has strongly demonstrated to be a tool that can be used to obtain the flow pattern for a given number of design-variables. One of the main applications of the CFD based optimisation is the shape-optimisation, especially in the aerodynamics field. The hydrodynamics of a surfboard on a wave define a complex system and could be difficult to analyze. Hendricks ${ }^{5}$ conducted one of the first studies on the surfboard hydrodynamics, where they demonstrated the existence of separating flow depending mostly on the surfboard fin geometry. Some examples of CFD studies upon different fin geometries and configurations are ${ }^{6-9}$. Brandner and Walker ${ }^{10}$ conducted an experimental study, investigating the flow around a surfboard fin, observing the lift, drag and moment variations as a function of the angle of attack at various Reynolds numbers to demonstrate that stall is a result of secondary flow effects and presented results for stall near the tip of the fin.

There has been substantial work in the area of surfboard fin shape optimisation ${ }^{1,8,9}$. Larvey and Carswell ${ }^{9}$ examined the hydrodynamics of the surfboard fins with the aim to understand the flow around the fins. They investigated several foils including NACA 4- and 6-series and conducted experiments involving various fin parameters. They also employed the use of fluid structure interaction (FSI) and CFD to investigate the flow around these fins and in order to develop an optimised shape of the fin. However, there is still a dearth of research in the optimisation techniques available or explored in this area. 
Consequently, works about aerodynamic shape optimisation were conducted and any useful information obtained was adapted to the particularities of the present study, which is a hydrodynamic optimisation of a surfboard fin.

A plethora of works have dealt with optimising the shape of flow devices for better aerodynamic performance, hence a few of them are going to be reported. Jeong et al. ${ }^{11}$ conducted two-dimensional airfoil shape optimisation, applying the Kriging model and using the PARSEC airfoil parametrisation and coupling Navier-Stokes code with genetic algorithms. The Kriging model is a response surface model, connecting the objective function with the design-variables using stochastic process. The first optimization was at a condition of Mach 2 and an angle of attack $(A \circ A) 2^{\circ}$, with constrained cross-sectional surface, such that it is identical with RAE2822. Painchaud-Ouellet et al. ${ }^{12}$ performed single-point and multi-point optimisation of two-dimensional airfoil design in transonic regime by using nonuniform rational B-splines parametrisation. A Navier-Stokes flow solver was used to obtain the aerodynamic coefficients. A design of experiments was conducted to reduce the number of the design-variables. The results showed that the nonuniform rational B-splines parameterisation resulted in smooth optimised airfoils and the multipoint optimisation resulted in airfoils with good efficiency at a specific Mach range. Chernukhin and Zingg ${ }^{13}$ studied multimodality in two- and threedimensional airfoil shape optimisation problems. The design-variables for the two-dimensional optimization were the $y$ coordinates of the B-spline control points, while the $x, y$ and $z$ coordinates of the B-spline control points were the design-variables of the three-dimensional optimization. The optimisation showed that none of the problems was highly multimodal and the majority of the two- and 
three-dimensional airfoil optimisation problems were unimodal. It must be mentioned, though, that three-dimensional optimisation problems, which included substantial geometric deformation, seemed to be somewhat multimodal. Epstein et al. ${ }^{14}$ applied various CFD-based optimisation techniques aiming to obtain an optimal three-dimensional wing shape, which presents the minimum drag. He used three different optimisers, namely SYN107 (Intelligent Aerodynamics Int'I), MDOPT (The Boeing Company) and OPTIMAS (Israel Aerospace Industries). A numerical solution of Navier-Stokes was performed using multigrid multiblock structured code FL017. The results were verified by running numerical simulations for the obtained optimal designs. All the aforementioned optimisation tools resulted in aerodynamically feasible shapes, which satisfied the defined constraints and presented less drag at all design points. Further CFD-based shape-optimisation studies can be found in other references ${ }^{15-18}$.

The major aim of the current work is to employ the genetic algorithms and Response Surface based Model (RSM) to present a technique for the surfboard fin shape optimisation. The study combines CFD simulations using the FLUENT ${ }^{\oplus}$ code with genetic algorithms to obtain an optimal fin shape, which presents maximum lift-to-drag ratio (L/D ratio). The objectives are summarised below:

- Design the experiments using the Latin Hypercube sampling technique.

- $\quad$ Perform CFD simulations on the different fin designs and construct a Response Surface based Model (RSM), which approximates the L/D ratio of each fin design. 
- $\quad$ Apply the genetic algorithm to the RSM to minimise drag-per-lift value and obtain the fin shape with maximum L/D ratio.

- Improve the RSM iteratively until an optimal fin shape is obtained.

- $\quad$ Perform CFD simulations on the optimal fin design and validate the improvement of L/D ratio along with an investigation of the flow field around the fin (lift and drag variation as a function of the angle of attack, stall point detection and flow visualisation).

\section{Methodology}

A fin design (initial fin), similar to the one used in Brandner and Walker ${ }^{10}$, was modelled initially to validate the reliability of the CAD model, the mesh and the CFD solver parameters setup. The main geometry characteristics of the initial fin are summarized in the Table 1. The validated initial fin design was then followed by the design of experiments using the Latin Hypercube sampling technique and the resultant fin designs, which explored efficiently the design space, were modelled. The next step was to construct the RSM using the lift and drag values obtained from the CFD simulations for the aforementioned fin designs. Finally, the RSM was optimised using genetic algorithms and was iteratively improved until an acceptable design was obtained. The CAD models of the fin designs and the corresponding control volumes surrounding them were created using CATIA ${ }^{\circledR}$ V5 software. 
The meshing of the models was performed using the ANSYS ${ }^{\circledR}$ Workbench meshing software. In the current work, an unstructured mesh was chosen due to the ease it offers during the meshing process as a number of different fin geometries were needed to be modelled. Refined mesh was used at the surface of the fin, and the meshes were constructed with the obtained $y+$ values of around 1. The inflation layer was then applied to the surface of the fin to capture the big velocity gradients that are induced by the boundary layer effects. Inflation is the generation of three-dimensional prism elements at selected surfaces to capture recirculation and other complex flow phenomena with acceptable accuracy at near-wall regions. The credibility of the mesh was confirmed by conducting a mesh sensitivity analysis, with respect to lift and drag coefficients for the initial fin design at various angles of attack with the convergence criterion of $10^{-6}$. It must be mentioned here that this analysis was carried out with half section of the fin.

\subsection{CFD Methodology}

The double-precision three-dimensional FLUENT ${ }^{\circledR}$ solver was used to solve the incompressible Reynolds Averaged Navier-Stokes (RANS) equations. The SST $k-\omega$ turbulence model was applied, as it is suitable for separating flows and adverse pressure gradients ${ }^{19}$. As far as the boundary conditions are concerned, the inlet of the control volume was defined as the velocity inlet, the outlet of the control volume was defined as the pressure outlet, the top and the side boundaries of the control volume were defined as symmetry and the fin as well as the bottom of the control volume (representing the surfboard) were defined as 
the wall. The velocity at the inlet was set to be $10 \mathrm{~m} / \mathrm{s}$ and was kept stable for all simulations, as the results in Brandner and Walker ${ }^{10}$ showed that the variation of Reynolds number in this case has negligible effects on the lift and drag coefficients. As the flow velocity is low enough not to cause compressibility ${ }^{20}$, hence the flow was considered incompressible. The working fluid was defined as liquid water with a density of $998.2 \mathrm{~kg} / \mathrm{m}^{3}$ and a viscosity of $0.001003 \mathrm{~kg} / \mathrm{ms}$. The SIMPLE scheme was chosen as the solution method with second-order spatial discretization. Finally, the standard initialization was used, and the solution was initialized by the values at the inlet of the control volume.

The vector of the design-variables for the present study consisted of six parameters, which defined the shape of the surfboard fin; these parameters are presented in Table 2. The hydrofoil was parameterized using the NACA four-digit parametrization. NACA four-digit airfoils are defined by three parameters, namely, the maximum camber as a percentage of the chord length, the maximum camber position as tens of a percentage of the chord length and the thickness as a percentage of the chord length. The design-variables are presented in Figure 1 (a) and (b), and the computational grid created for this geometry is shown in the Figures 2, 3 and 4 along with the domain dimensions.

The design space is the area bounded by the upper and lower limits of the designvariables. The upper and lower limits of the maximum camber, maximum camber position and thickness were defined according to the NACA airfoil parametrization. The upper and lower limits of the chord length, the depth and the sweep angle were defined after conducting research upon various commercial surfboard fin designs, in order to avoid any unrealistic designs. 


\subsection{Latin Hypercube Sampling}

The design of experiments was performed using the Latin Hypercube Sampling technique. Latin Hypercube Sampling is a statistical technique, used for obtaining a set of feasible values for the design-variables from a multidimensional distribution and is widely used in computational experiments. Further information regarding Latin Hypercube Sampling technique can be found $\mathrm{in}^{21}$. For the construction of the Latin Hypercube Sample, the function "Ihsdesign" provided by the statistics and machine learning toolbox of MATLAB ${ }^{\circledR}$ was used. The version of the function, which was used, iteratively generates Latin Hypercube Samples to find the best one according to the "maximin criterion" (maximizes minimum distance between points). The resultant Latin Hypercube Sample consisted of 42 different fin designs.

\subsection{Response Surface based Model (RSM)}

A quadratic regression polynomial was used to approximate the response of the system. Polynomial regression is a model, which can be used to approximate non-linear responses ${ }^{22}$. The general form for polynomial regression is

$$
y=\sum_{i=0}^{k} \beta_{i} \cdot x^{i}
$$

where $y$ is the response of the system, $x$ the design-variables vector and $\beta_{i}$ are the regression coefficients. The general form of a quadratic regression polynomial, which approximates a multivariate function, is 


$$
y=\beta_{0}+\sum_{i=1}^{n} \beta_{\mathrm{l}} \cdot x_{I}+\sum_{j=1}^{n} \beta_{j j} \cdot x_{j}^{2}+\sum_{i=1}^{n-1} \sum_{j=i+1}^{n} \beta_{i j} \cdot x_{i} \cdot x_{j}
$$

The number of regression coefficients $(\mathrm{N})$ is equal to

$$
N=\frac{(n+1) \cdot(n+2)}{2}
$$

where $\mathrm{n}$ is the number of the design-variables. The number of design-variables for the present case is six; hence, the regression polynomial included 28 regression coefficients. After conducting the CFD simulations, an overdetermined system was formulated with the unknown regression coefficients. This system was solved by least-squares fitting using the "mldivide" command of $M_{A T L A B}{ }^{\circledR}$ to obtain the regression coefficients and the formula, which approximates the response of the system. The aim of the present study is to maximize the $L / D$ ratio through the system's response of drag-per-lift values obtained through the CFD simulations. This is achieved as the optimization algorithms search for the minimum of the drag-per-lift function. The objective function of the optimisation was the resultant polynomial which represented the variation of the drag-per-lift ratio as function of the design-variables. The optimization process minimized the drag-per-lift; hence, the $L / D$ ratio became maximum, which was the principal aim of the present study. The operating point, at which the surfboard fin was optimized, was at a velocity of $10 \mathrm{~m} / \mathrm{s}$ and angle of attack $10^{\circ}$. This angle of attack was chosen to ensure that all fin designs were evaluated before stall point, as after stall point CFD accuracy is limited due to the separation of flow and the fact that the results come from averaging. The process 
flow chart for the Response Surface based optimization procedure is shown in the Figure 5.

\subsection{Optimisation using Genetic Algorithms}

The resultant RSM was optimized using the genetic algorithm, provided within the MATLAB ${ }^{\circledR}$ optimisation toolbox. Genetic algorithms are a subcategory of evolutionary algorithms, and the main concept, on which they are based, is to generate a set of possible solutions for a specific problem. In computer science, the procedure of searching the best solution for a given problem among multiple possible solutions is known as searching for the search space and the set of the possible solutions as the search space. Chromosome corresponds to a point in the search space. The genetic algorithm evaluate populations of chromosomes and iteratively transitions from one population to another. Another main element of a genetic algorithm is the fitness function, based on which every chromosome in a specific population is assessed and gets a corresponding score (fitness). Fitness expresses how efficiently the corresponding chromosome resolves the given problem. Further information about the genetic algorithms can be found $\mathrm{in}^{23}$. The constructed RSM was defined as the fitness function of the optimisation problem. The constraints were defined as presented in Table 3. It must be mentioned that the last constraint is non-smooth and that is the main reason the genetic algorithm was chosen to solve the present problem, as the other optimization algorithms provided by $\operatorname{MATLAB}^{\circledR}$ were not able to handle such a 
constraint. The final optimal design resulted after improving iteratively the RSM. The basic steps of the process are summarized below:

- Optimal design obtained applying the optimisation algorithm to the RSM

- $\quad$ CFD analysis for the optimal design was performed and the response of the system was obtained

- The values of the design-variables for the optimal design were added to the set of samples of the RSM

- The response was added to the set of responses of the RSM

- The process stopped when the value obtained solving the optimisation problem differed within an acceptable tolerance in comparison with the value obtained from the numerical analysis.

\section{$3 \quad$ Results and Discussion}

Lift and drag forces were non-dimensionalised as:

$$
\begin{aligned}
& C_{L}=\frac{L}{\frac{1}{2} \cdot \rho \cdot v^{2} \cdot A} \\
& C_{D}=\frac{D}{\frac{1}{2} \cdot \rho \cdot v^{2} \cdot A}
\end{aligned}
$$

where $L$ is the lift force, $D$ is the drag force, $\rho$ is the fluid density $\left(998.2 \mathrm{~kg} / \mathrm{m}^{2}\right), u$ is the fluid velocity $(10 \mathrm{~m} / \mathrm{s})$ and $A$ is the planform area of the fin $\left(0.00962 \mathrm{~m}^{2}\right)$. 
Three meshes were used to perform the mesh sensitivity study for the initial design namely coarse (0.6 Million elements), medium (1 Million elements) and fine (1.4 Million elements) grids. The results of the mesh sensitivity study are presented in Table 4. The results show that the medium mesh capture with sufficient accuracy the lift and drag values as the difference between the obtained values using the medium and fine meshes are negligible. The coarse mesh successfully obtained the drag value but the lift value differs substantially in comparison with the values obtained using the medium and fine meshes. Consequently, the medium mesh was used for the present study and the convergence criteria was set to of $10^{-6}$ for all cases.

Pressure contours and velocity contours at the plane parallel to the bottom of the control volume and lies $0.05 \mathrm{~m}$ above it, are presented in the Figure 6 . The pressure and velocity contours are in accordance with each other, because in areas with high velocities (upper and lower surface of the foil) low pressures are noticed, while at the leading and trailing edge, which are low-velocity areas, high pressures are observed. Moreover, the optimal fin has an efficient trailing edge of foil, as low pressures are not present at the rear part of the fin. Flow visualisations demonstrate that for the $A \circ A=0^{\circ}$ the flow is uniform and stable around the fin, which is expected as the hydrofoil is symmetrical. As the AoA increases, vortices are noticed near the tip of the fin which are gradually increasing with respect to size and intensity as shown near the tip of the fin in the Figure 7 . Further, the velocity contours at planes parallel to the inlet of the control volume for AoA $18^{\circ}$ are presented in the Figure 8. Flow separation is noticed at the third and the fourth plane (right to left) near the tip of the fin; hence these 
contours are in accordance with the aforementioned assumption, that the stall point is detected at $18^{\circ}$.

The lift and drag coefficient variations as function of angle of attack are compared with the corresponding variation obtained in Brandner and Walker ${ }^{8}$ and the comparison is illustrated in the Figure 9. Drag coefficient variation is almost coincident for the two studies, while the curves representing the lift-coefficient variation present minor differences until an angle of attack $18^{\circ}$. After this angle of attack, the curves follow different trends. For the present study, the stall point is detected at $18^{\circ}$ and it is under-predicted in comparison with the study of Brandner and Walker, in which the stall point was detected at $22^{\circ}$. The differences after the stall point could be a result of the limited accuracy of the CFD analysis at this region. Consequently, the created model is considered reliable for an angle of attack smaller than the stall point.

The same meshing and CFD setup parameters (number of inflation layers, first layer thickness and size of elements) were used for simulating the fin designs of the Latin Hypercube Sample for angle of attack $10^{\circ}$. The geometric characteristics of the optimal fin design, which resulted from the optimization process, are presented in Table 5 which is the result obtained after nine iterative improvements. Table 6 presents the results of each iterative process for all the parameters in consideration.

The observed L/D ratio for the optimal design at an angle of attack $10^{\circ}$ was 14.32 , while the $L / D$ ratio of 8.81 was observed for the initial design at the same angle of attack. This increase in the L/D ratio indicates an overall improvement of 
approximately $62 \%$ which is a considerable improvement in the design. The difference between the drag-per-lift value obtained from the optimisation solver and the corresponding one obtained from CFD analysis indicates the error of the RSM predicting the drag-per-lift value of a fin design compared with the real value obtained from the CFD solver. The RSM error is presented as a percentage for the nine iterations in the chart of Figure 10. According to the aforementioned chart, the RSM is gradually improving until the $9^{\text {th }}$ iteration, when the RSM error was $5.28 \%$, which was considered an acceptable tolerance for the present study. There are mainly two indicators of the efficiency of an RSM, namely the adjusted root-mean-square error and the adjusted coefficient of multiple determination. For the improved RSM, the adjusted root-mean-square error was found to be 0.0063 , which is relatively small compared with the drag-per-lift ratio values; hence the RSM is considered efficient based on the root-mean-square error. The adjusted coefficient of multiple determination was found to be 0.9504 , which is considered close enough to 1 , indicating the efficiency of the RSM.

Figure 11 represents the lift and drag coefficients variations for the optimal design as a function of the angle of attack. The plot demonstrates that the lift and drag variations are in accordance with the established knowledge, as drag increases along with an angle of attack, while lift increases linearly up to the stall point and then drops. The stall point was detected at angle of attack $18^{\circ}$. A further comparison of lift per drag ratio of the two designs (initial and optimal) for more angles of attack was performed as demonstrated in the Figure 12. The comparison showed that while the initial design performs better for small angles of attack $\left(0^{\circ}\right.$ and $\left.4^{\circ}\right)$, it becomes inefficient for angles higher than $4^{\circ}$. It can be 
clearly seen that the L/D curve drops very quickly after $4^{\circ} \mathrm{AoA}$. On the other hand, the optimal design performs much better for the angles of attack larger than $4^{\circ}$. The aforementioned behavior is justified by the fact that the hydrofoil of the optimal design is symmetrical, while the one of the initial shape is a half section and non-symmetrical hydrofoils generate substantial lift even for small angles of attack.

\section{Conclusions}

The result of the optimisation was a high aspect ratio, medium swept design with almost symmetrical hydrofoil. The drag-per-lift ratio values obtained from the optimisation and the CFD solver differed by $5.26 \%$. An overall improvement of approximately $62 \%$ has been obtained for the optimal design of the surfboard fin, where the optimal design presented the maximum L/D ratio for an angle of attack of $10^{\circ}$ among the forty-two designs of the Latin Hypercube Sample. Taking into account the lift coefficient variation of the optimal design as function of the angle of attack, the stall point was detected at $18^{\circ}$. The comparison of the optimal design with the initial design for angles of attack from $0^{\circ}$ to $20^{\circ}$ showed that the initial design is more efficient for an angle of attack smaller than $4^{\circ}$, while optimal design is more efficient beyond this angle of attack. This is attributed to the fact that the initial design is a half section hydrofoil, while the optimal design is a symmetrical hydrofoil. The visualization of the flow around the optimal fin confirmed the existence of flow separation at angle of attack $18^{\circ}$ near the tip of the fin. The RSM based methodology (along with iterative improvements) produce satisfactory results, as the resultant fin design has similar characteristics to commercial single fins, namely big depth, high aspect ratio, symmetrical 
hydrofoil and medium sweep angle. However, there is a lot of scope for improvements in the optimisation technique applied in the present study. Some indicative improvements could be the sensitivity study of the design-variables as well as the multimodal optimisation by replacing the non-smooth constraint and using more optimisation algorithms (e.g. multistart/singlestart SQP). 


\section{References:}

1. Lavery, N., Foster, G., Carswell, D. and Brown, S. Optimization of Surfboard Fin Design for Minimum Drag by Computational Fluid Dynamics (Do Glass-on Fins Induce Less Drag than Boxed Fins?). $4^{\text {th }}$ International Surfing Reef Symposium, 2005.

2. McTavish, B. A Personal History of Surfboard Design. Tracks Magazine, 3, 1973.

3. Carroll, N. The Next Wave: A Survey of World Surfing. Queen Anne Press, London, 1991.

4. Simpson, D., Fin Sciences, Surfboard fin lift, drag, hold and drive, [Online Resource]. Available: http://www.finsciences.com/surfboard-fin-lift-draghold-drive [Accessed: $10^{\text {th }}$ July 2015]

5. T. Hendricks, "Surfboard hydrodynamics, Part I: Drag; Part II: Pressure; Part III: Flow Separation," Surfer Magazine, 1969.

6. Carswell, D., Lavery, N., and Brown, S., Computational Modelling of Surfboard Fins for Enhanced Performance. The Engineering of Sport 6, 425-430, 2006, Springer New York.

7. Gudimetla, P., Kelson,N., and El-Atm, B. Analysis of the hydrodynamic performance of three- and four-fin surfboards using computational fluid dynamics. Australian Journal of Mechanical Engineering, vol. 7, no. 1, pp. 61-67, 2009.

8. Carswell, D. and Lavery, N., 3D solid fin model construction from 2D shapes using non-uniform rational B-spline surfaces, Advances in Engineering Software, 37, 8, 491-501, 2006, Elsevier. 
9. Carswell, D. Hydrodynamics of Surfboard Fins, PhD Thesis, University of Swansea, 2007.

10. Brandner, P. and Walker, G. Hydrodynamic performance of surfboard fin. The Fifteenth Australasian Fluid Mechanics Conference, Sydney, 2004.

11. Jeong, S., Murayama, M. and Yamamoto, K. Efficient Optimization Design Method Using Kriging Model. Journal of Aircraft, vol. 42, no. 2, pp. 413420, 2005.

12. Painchaud-Ouellet, S. et al. Airfoil Shape Optimization Using a Nonuniform Rational B-Splines Parameterization Under Thickness Constraint. AAIA Journal, vol. 44, no. 10, pp. 2170-2178, 2006.

13. Chernukhin, O. and Zingg, D. Multimodality and Global Optimization in Aerodynamic Design. AAIA Journal, vol. 51, no. 6, pp. 1342-1354, 2013.

14. Epstein, B. et al. Comparative Study of Three-Dimensional Wing Drag Minimization by Different Optimization Techniques. Journal of Aircraft, 462, pp. 526-541, 2009.

15. Bray-Miners, J. et al. Biomechanics of Slalom Water Skiing. Proc IMechE Part P: J Sports Engineering and Technology, 229(1) 47-57, 2015.

16. Luque, P. et al. Racing car chassis optimization using the finite element method, multi-body dynamic simulation and data acquisition. Proc IMechE Part P: J Sports Engineering and Technology, 227(1) 3-11, 2012.

17. Stier, B. et al. Analysis, manufacturing, testing, and structural optimization of a novel composite kiteboard design. Proc IMechE Part P: J Sports Engineering and Technology, 229(4) 248-265, 2015. 
18. Banks, J. et al. Kayak blade-hull interactions: A body force approach for self-propelled simulations. Proc IMechE Part P: J Sports Engineering and Technology, 228(1) 49-60, 2014.

19. Menter, FR. Two-Equation Eddy-Viscosity Turbulence Models for Engineering Applications. AIAA Journal. 32, 8, pp. 1598-1605, 1994.

20. Jinping, LI et al. CFD numerical simulation of water hammer in pipeline based on the Navier-Stokes equations. ECCOMAS, Lisbon, Portugal, 1417 June 2010.

21. Queipo, NV. et al. Surrogate-Based Analysis and Optimization. Progress in Aerospace Sciences, 41, 1, pp. 1-28, 2005.

22. Gatignon, H. Statistical Analysis of Management Data, 2nd ed., New York: Springer Publication, 2010.

23. Mitchell, M. An Introduction to Genetic Algorithms, The MIT Press, 1998.

24. Cantrell, P., Airfoils, [Online Resource]. Available: http://www.copters.com/aero/airfoils.html [Accessed: $5^{\text {th }}$ March 2017] 


\section{List of Figures:}

Figure 1. Design-Variables, (a) Fin, (b) Hydrofoil24.

Figure 2. Computational Domain used for the Simulation along with the Dimensions.

Figure 3. Grid around the surfboard fin, XY-plane.

Figure 4. Grid around the surfboard fin, YZ-plane.

Figure 5. Flow chart for the Response surface based optimization procedure.

Figure 6. Pressure (top) and Velocity (bottom) contours for Optimal Design $\mathrm{AOA}=0^{\circ}$.

Figure 7. Velocity Streamlines for Optimal Design at various AoA.

Figure 8. Velocity Contours Optimal Design, $A \circ A=18^{\circ}$

Figure 9. Lift and Drag coefficients vs. Angle of attack, comparison with Brandner \& Walker ${ }^{10}$.

Figure 10. RSM Error as function of Iterations

Figure 11. Lift and Drag vs. Angle of attack (Optimal Design).

Figure 12. L/D Ratio vs. Angle of Attack, Comparison of Initial and Optimal Designs. 


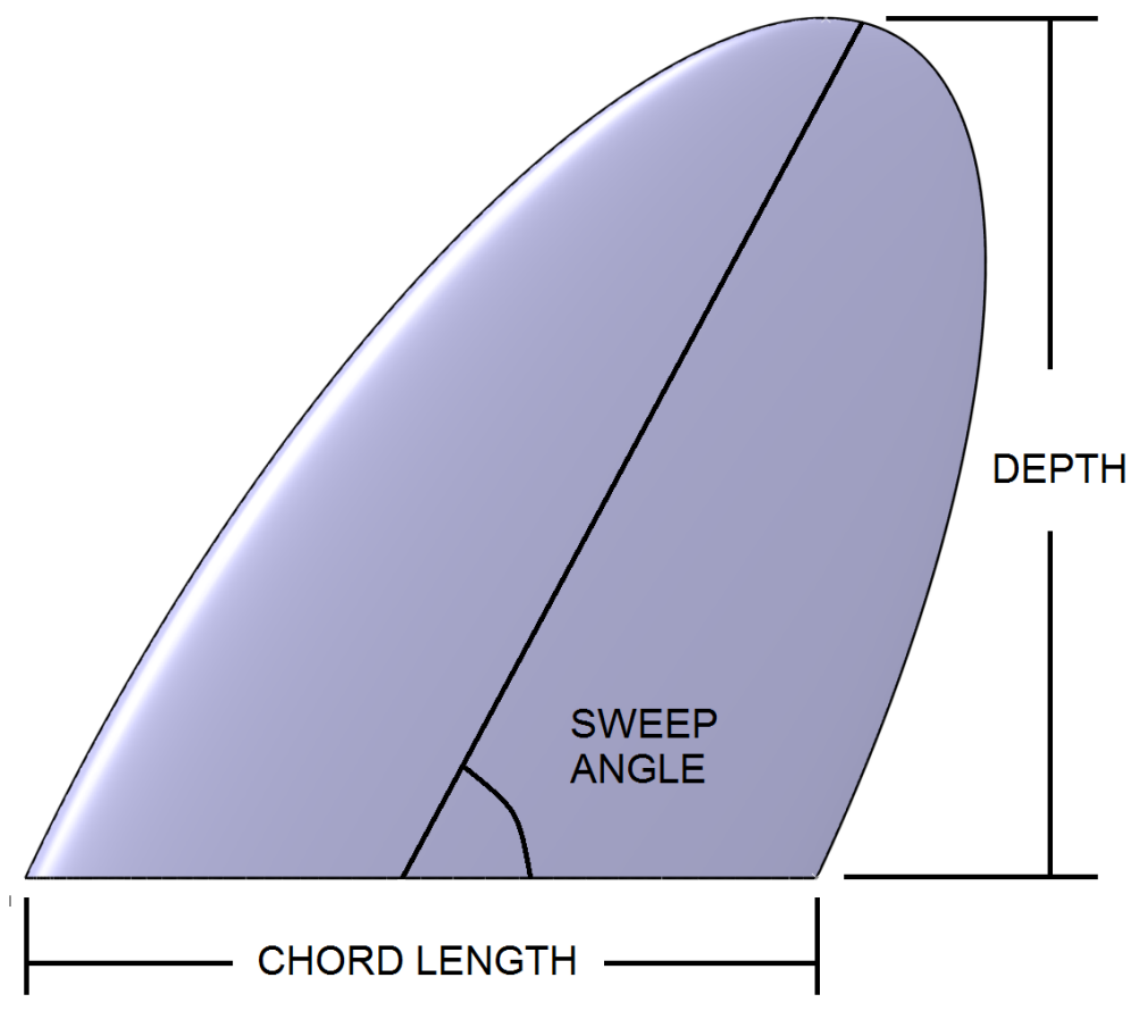

(a)

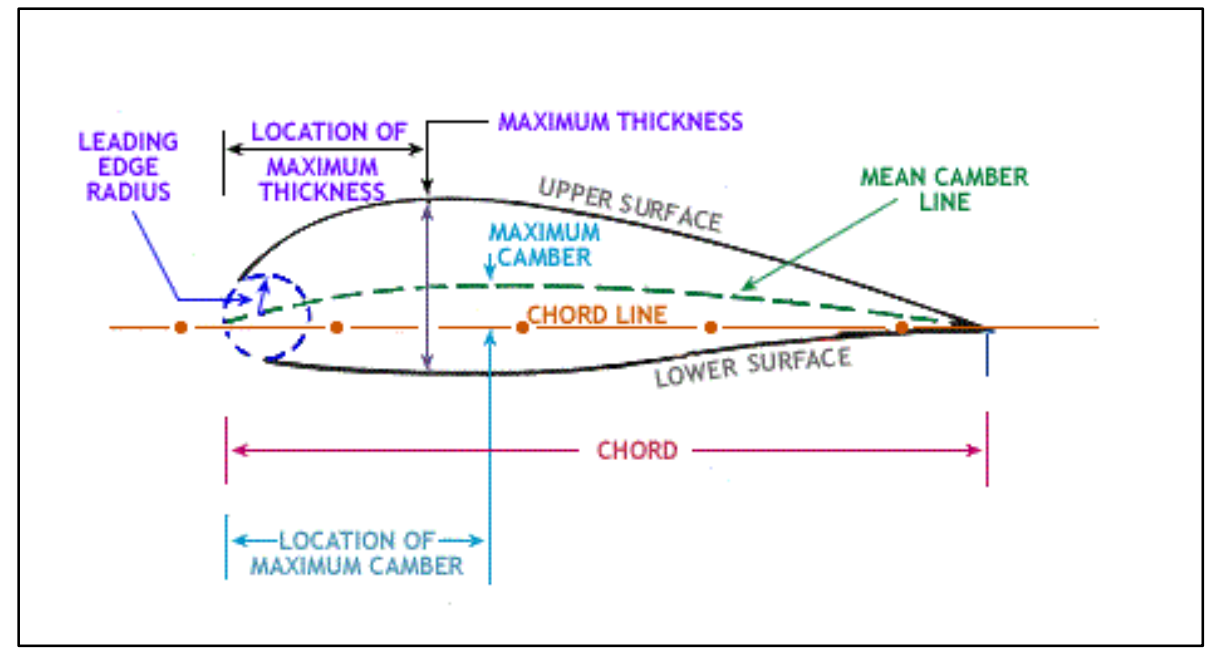

(b)

Figure 1. Design-Variables, (a) Fin, (b) Hydrofoil24. 


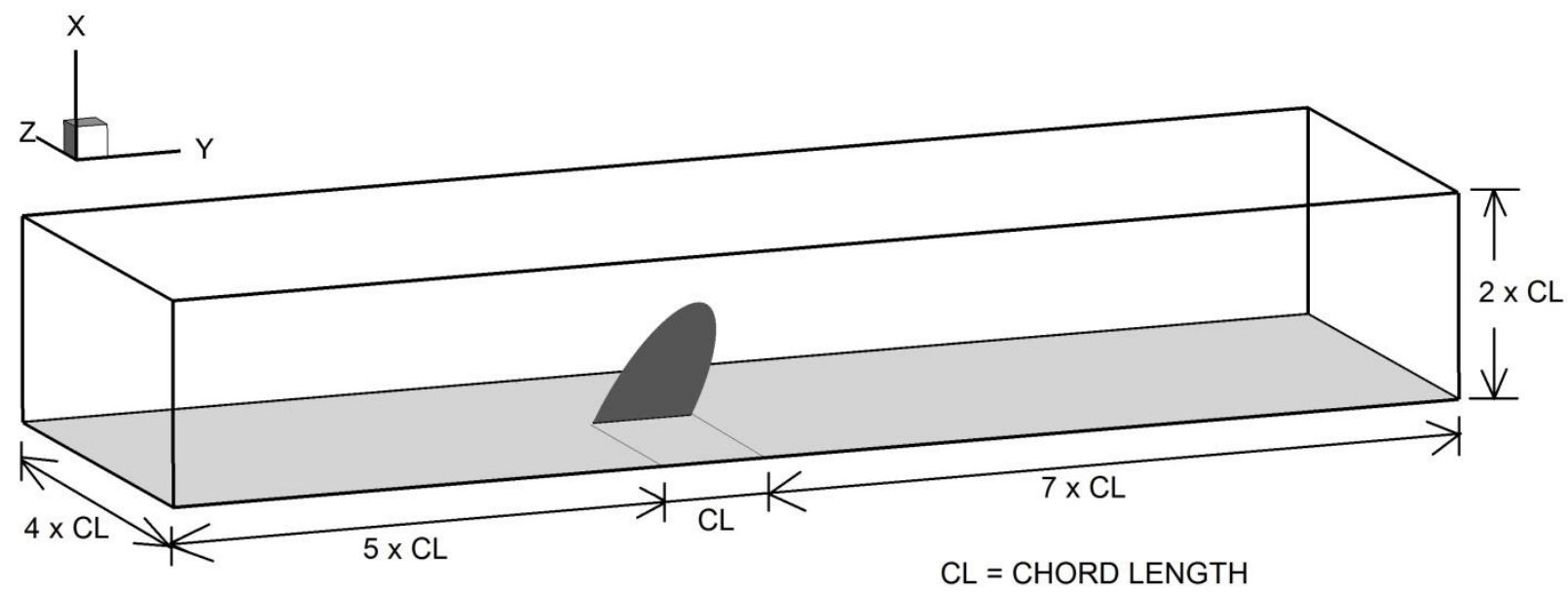

Figure 2. Computational Domain used for the Simulation along with the Dimensions. 


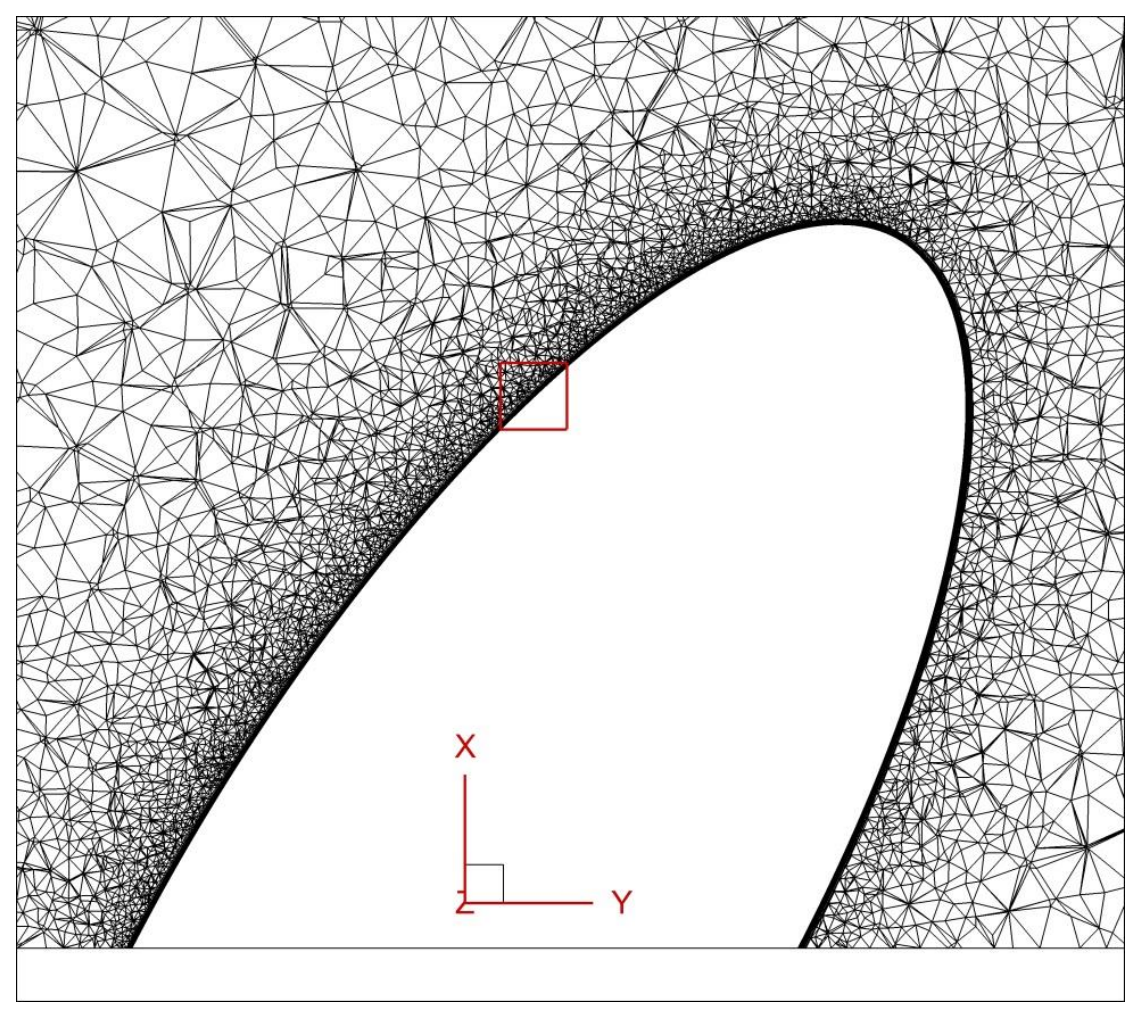

(a)

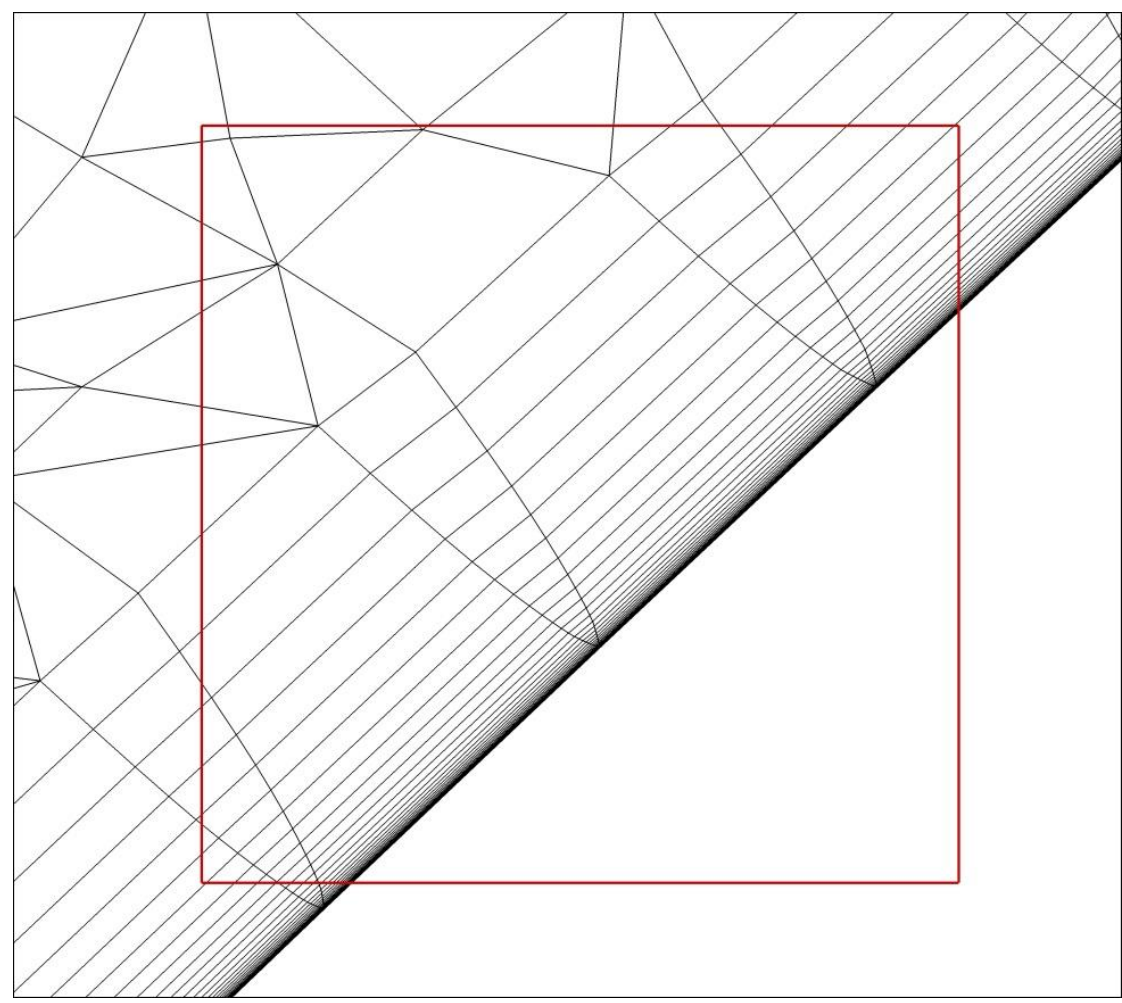

(b)

Figure 3. Grid around the surfboard fin, $X Y$-plane. 


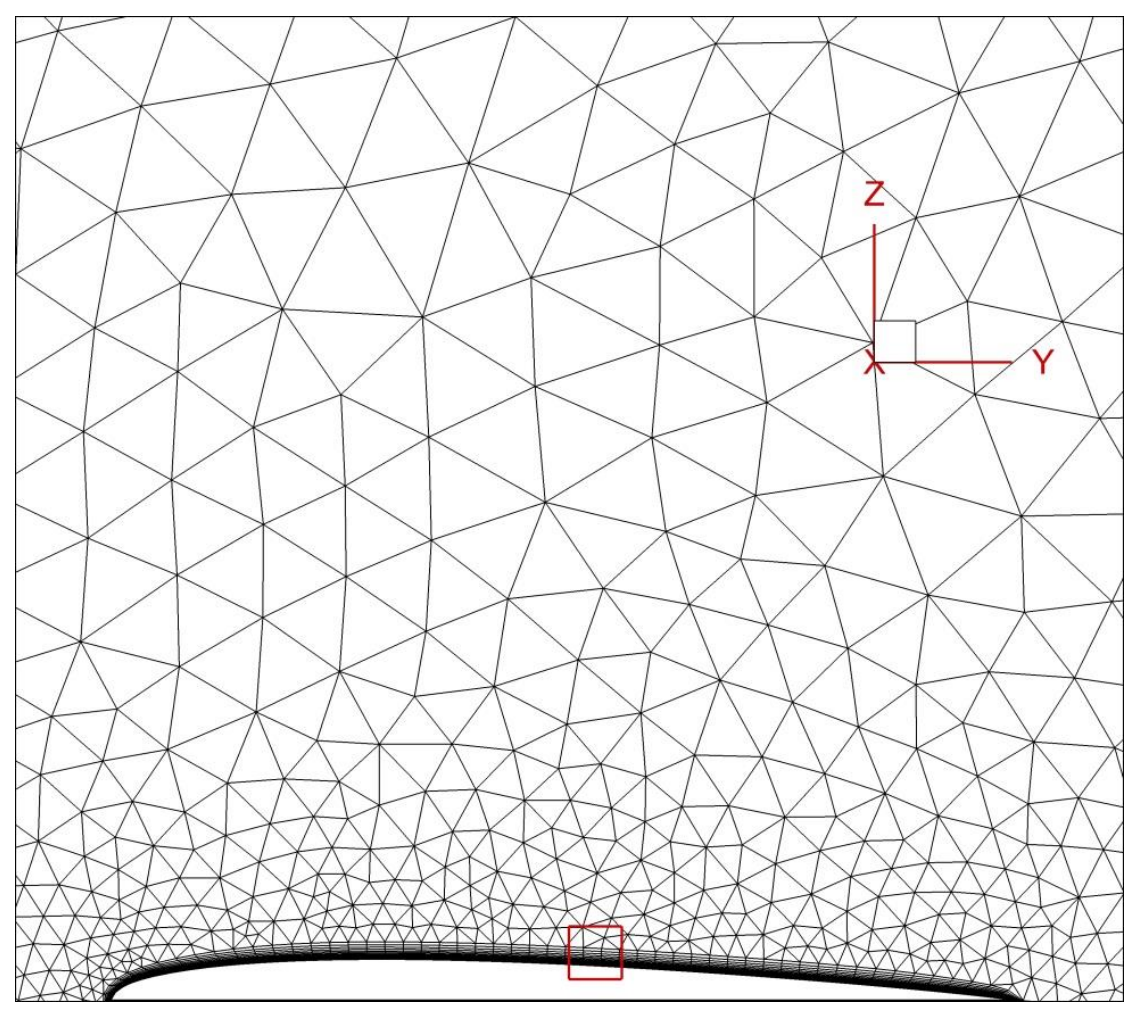

(a)

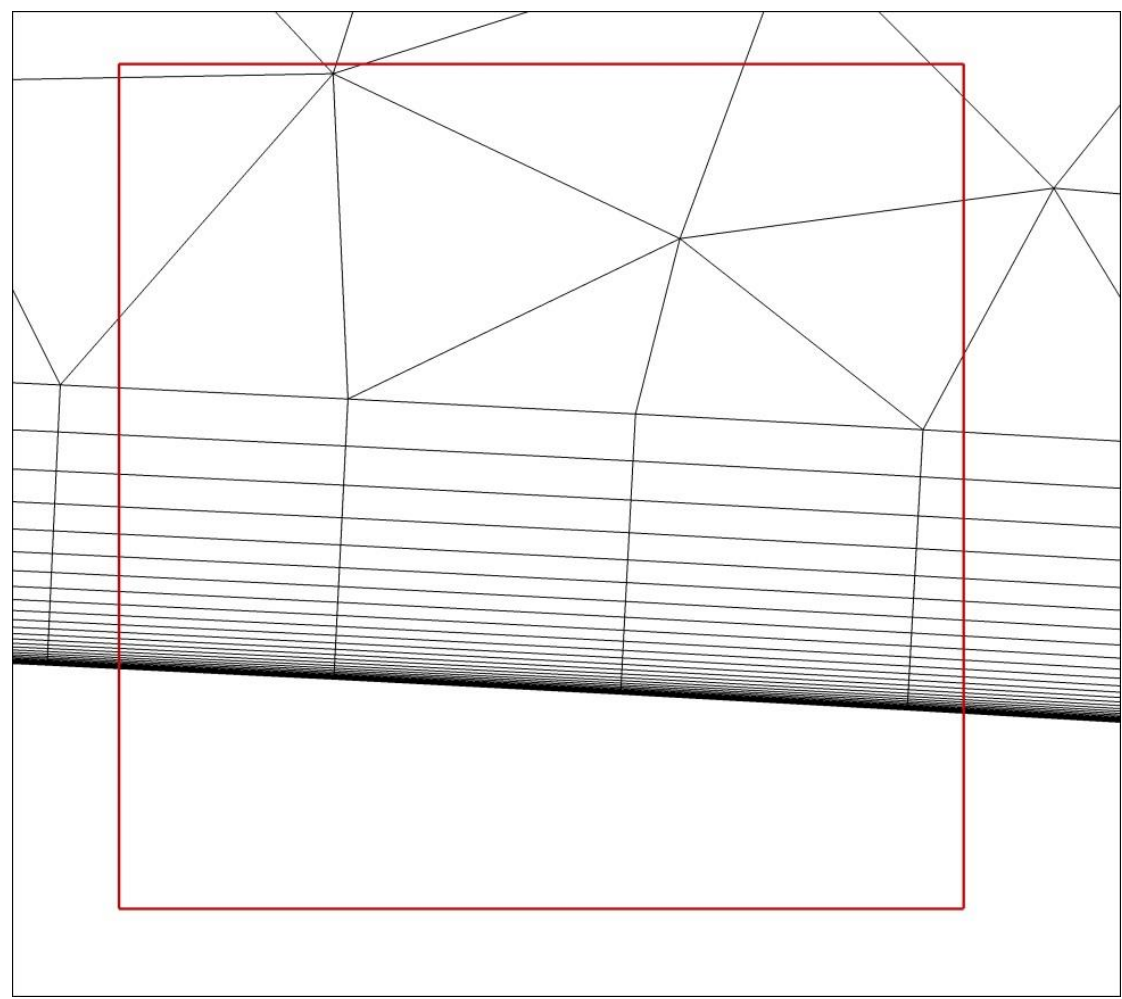

(b)

Figure 4. Grid around the surfboard fin, YZ-plane. 


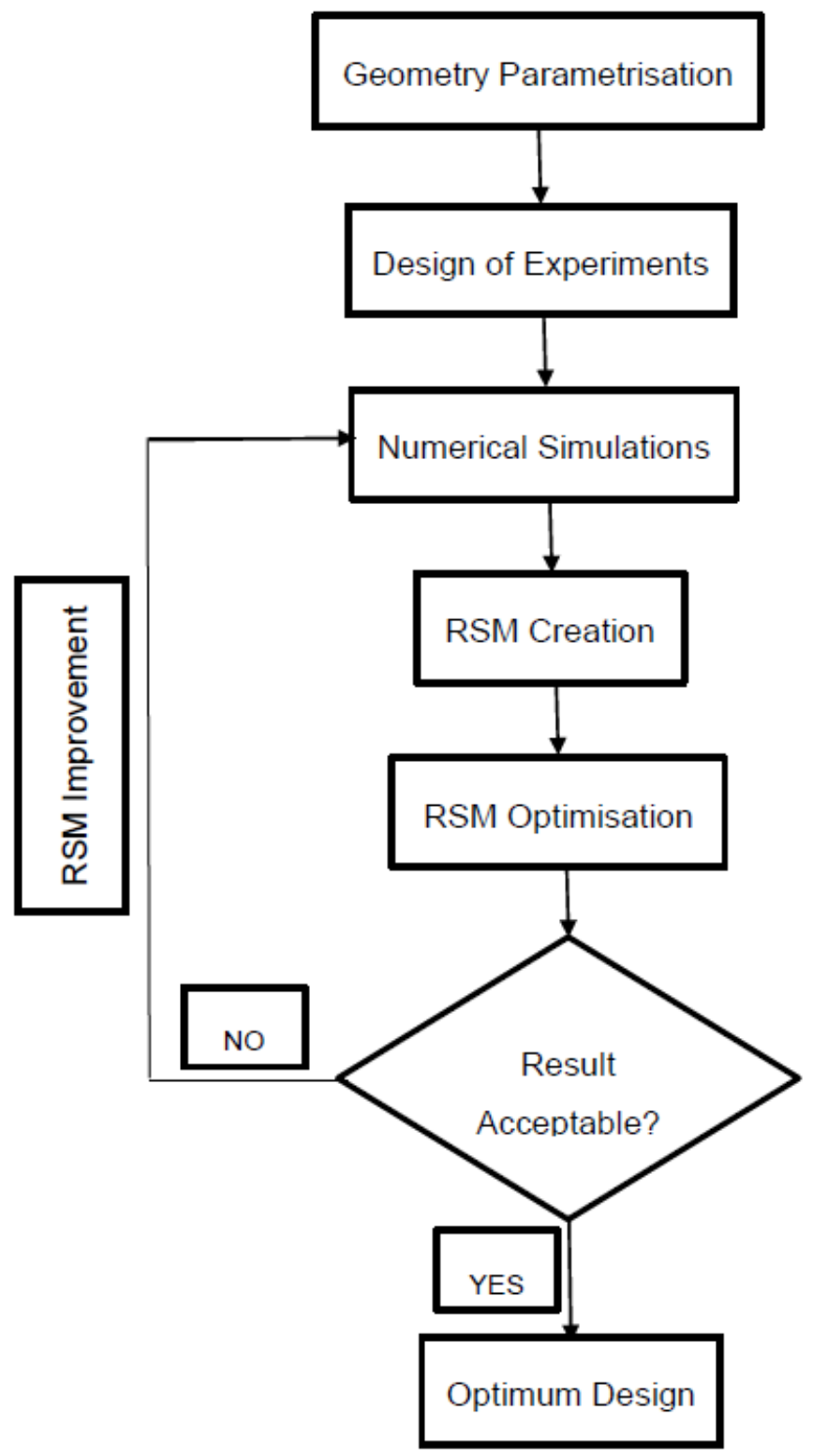

Figure 5. Flow chart for the Response surface based optimization procedure. 

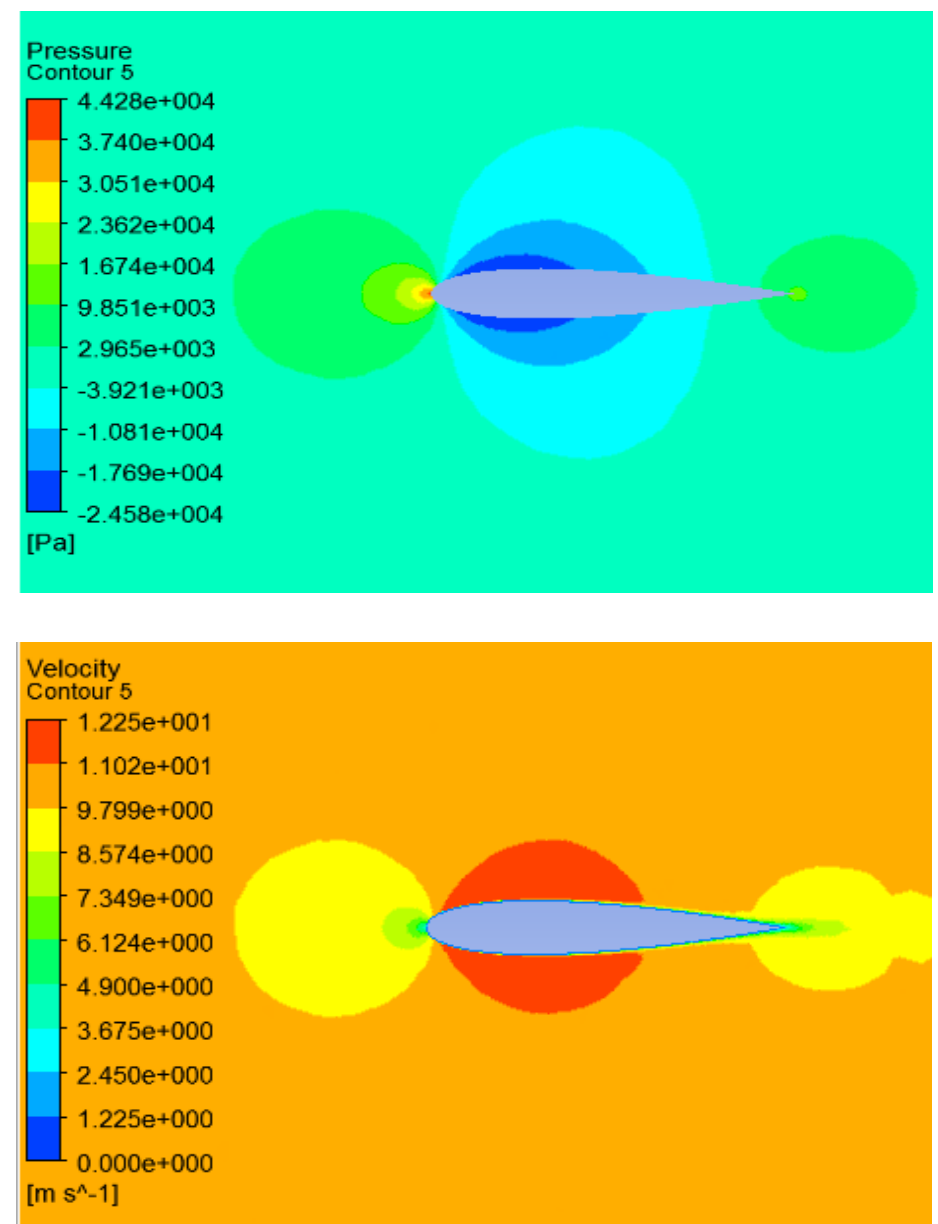

Figure 6. Pressure (top) and Velocity (bottom) contours for Optimal Design $A O A=0$. 


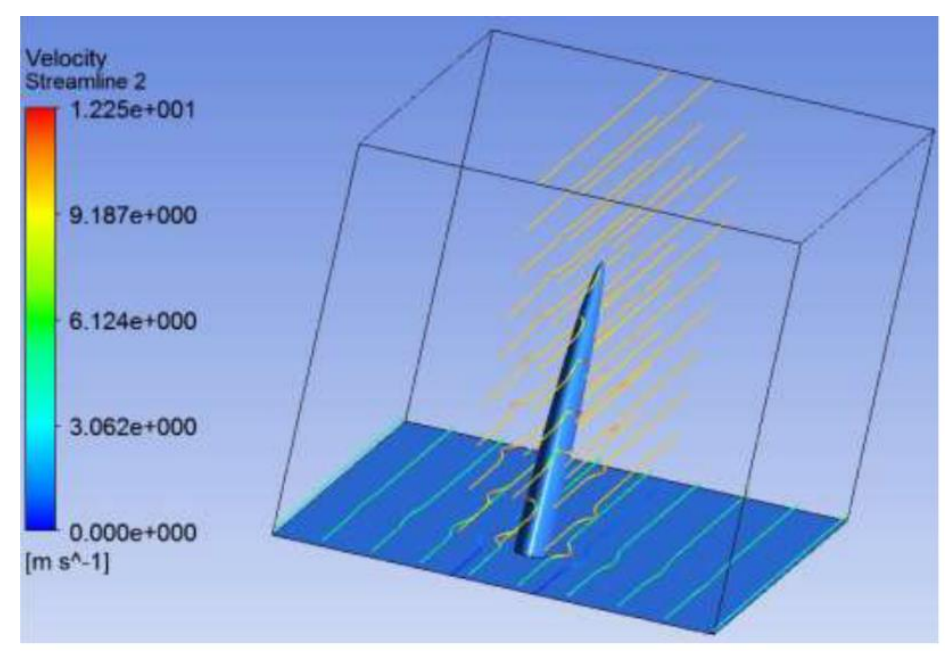

$$
A \circ A=0
$$

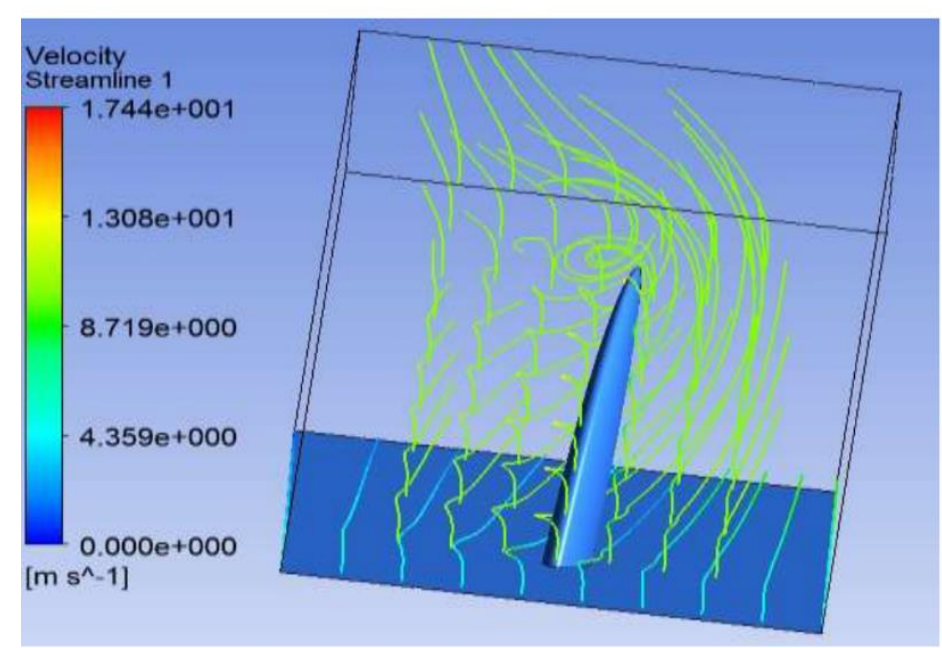

$$
\mathrm{A} \circ \mathrm{A}=8
$$

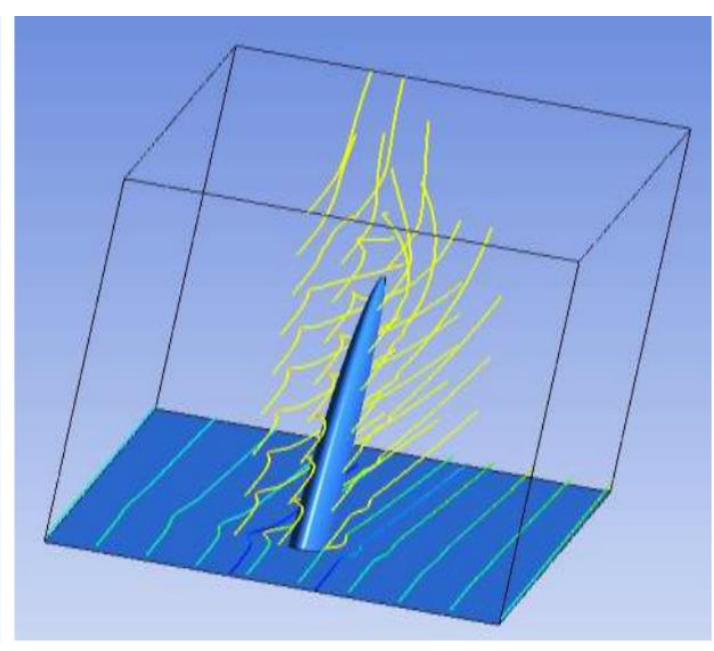

$\mathrm{A} \circ \mathrm{A}=4$

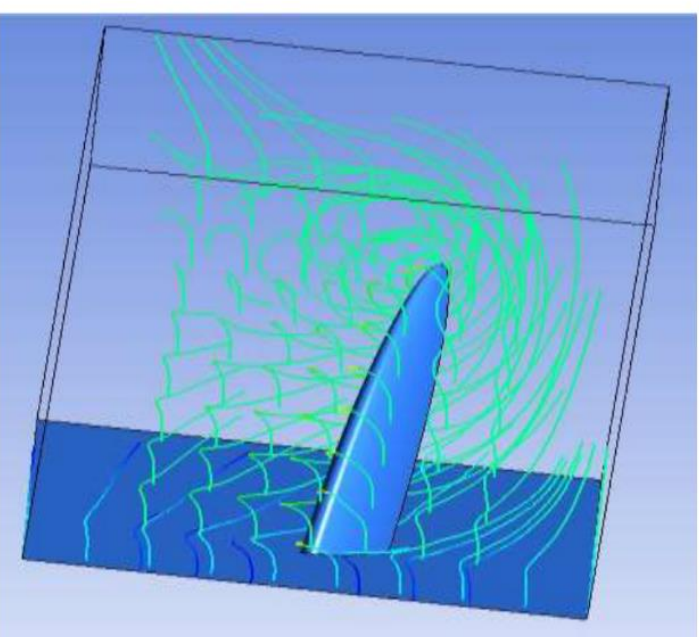

$\mathrm{A} \circ \mathrm{A}=18$

Figure 7. Velocity Streamlines for Optimal Design at various AoA. 


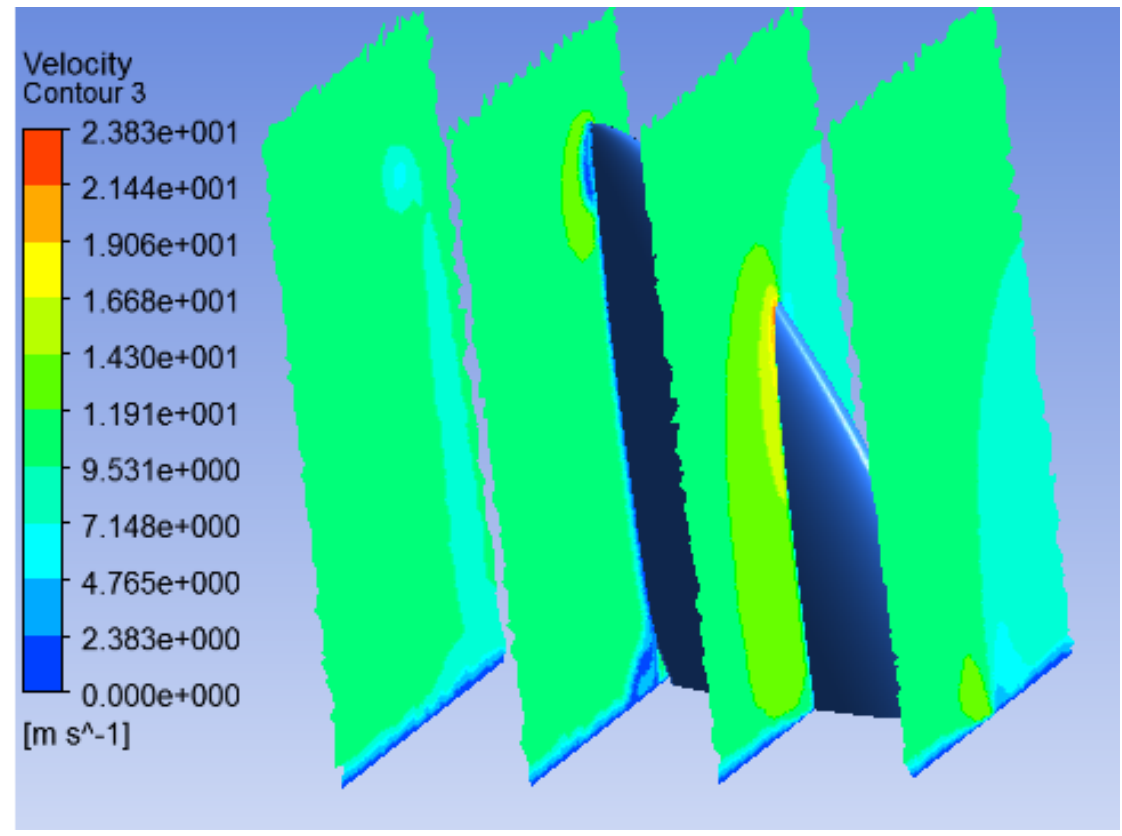

Figure 8. Velocity Contours Optimal Design, $A \circ A=18^{\circ}$ 


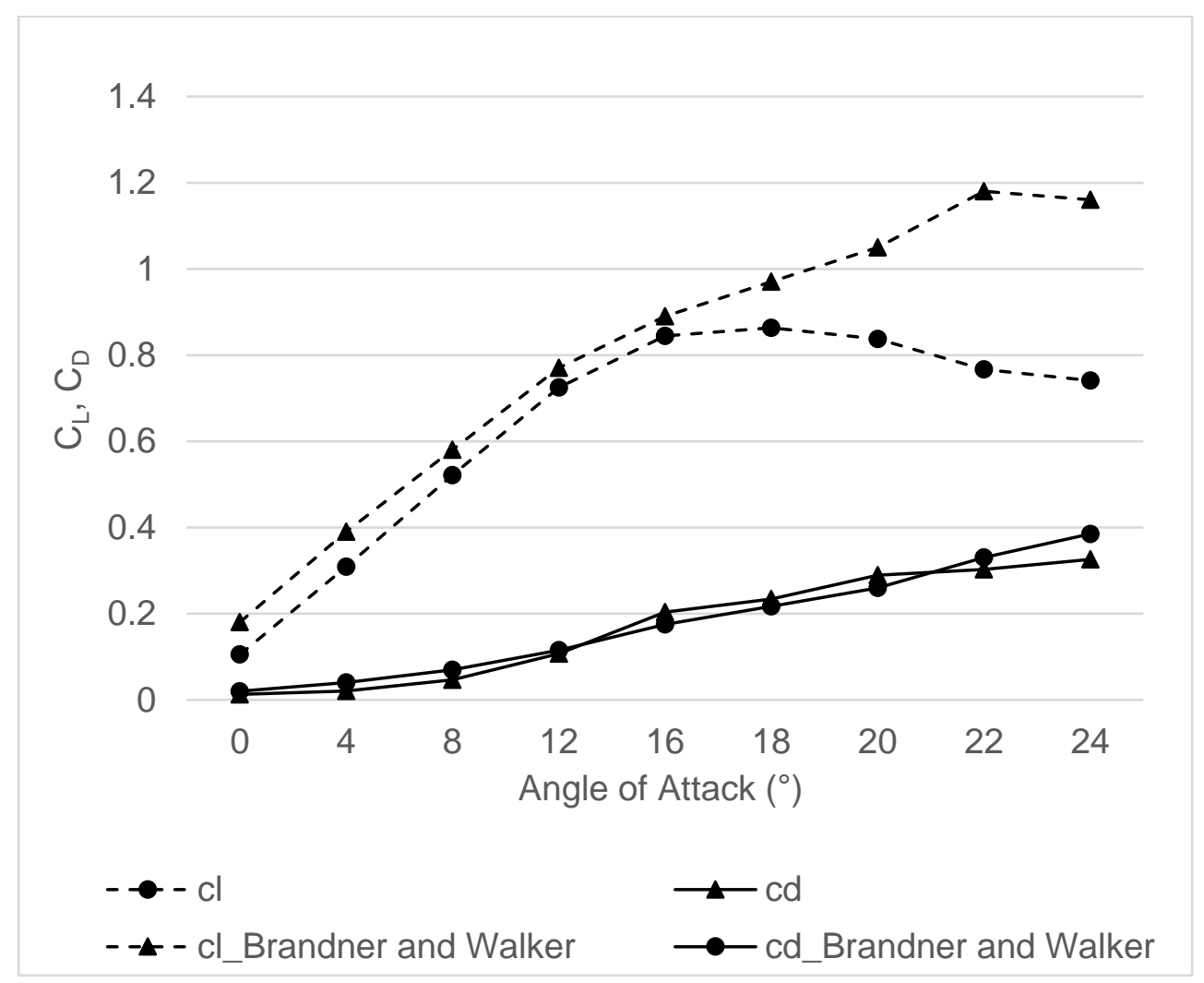

Figure 9. Lift and Drag coefficients vs. Angle of attack, comparison with Brandner \& Walker ${ }^{10}$. 


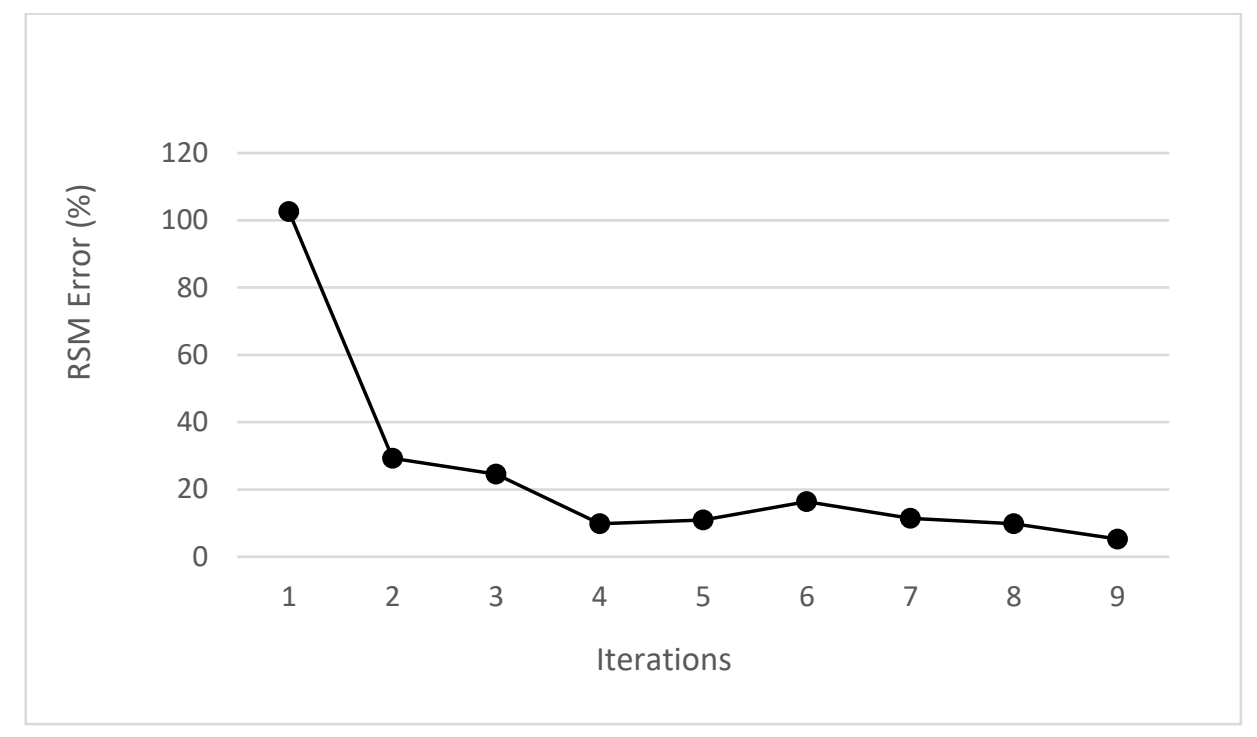

Figure 10. RSM Error as function of Iterations 


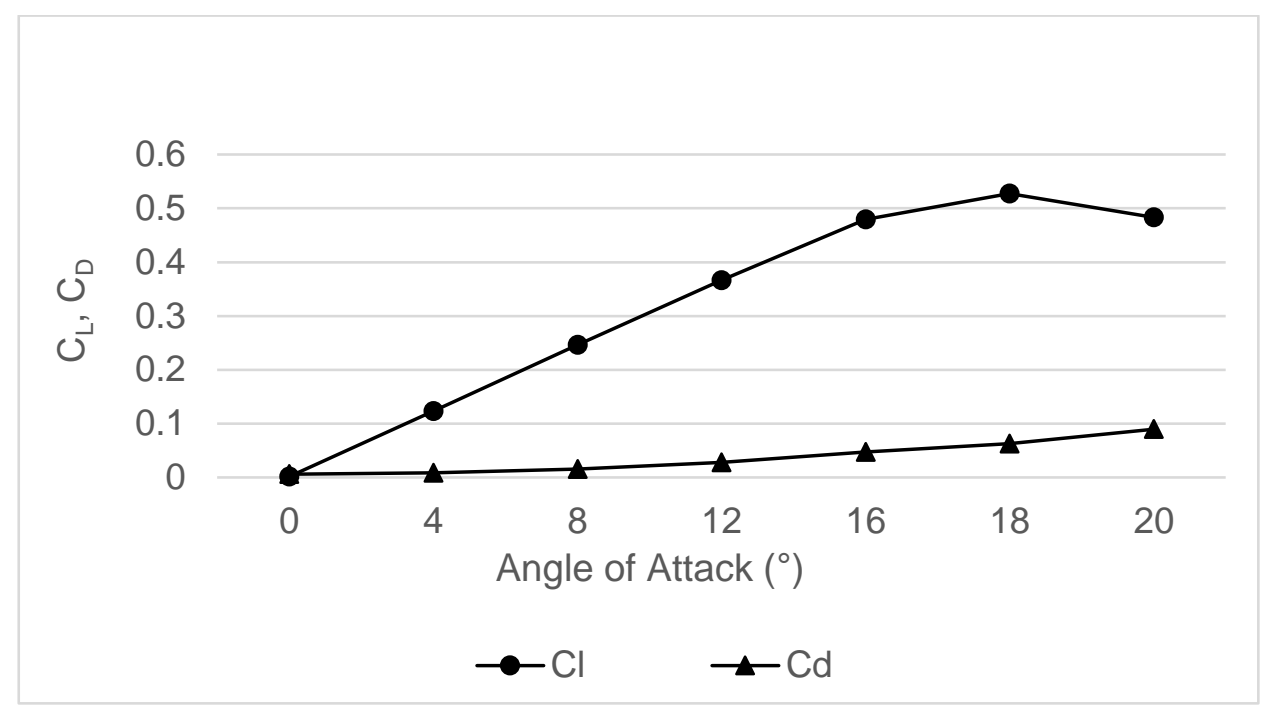

Figure 11. Lift and Drag vs. Angle of attack (Optimal Design). 


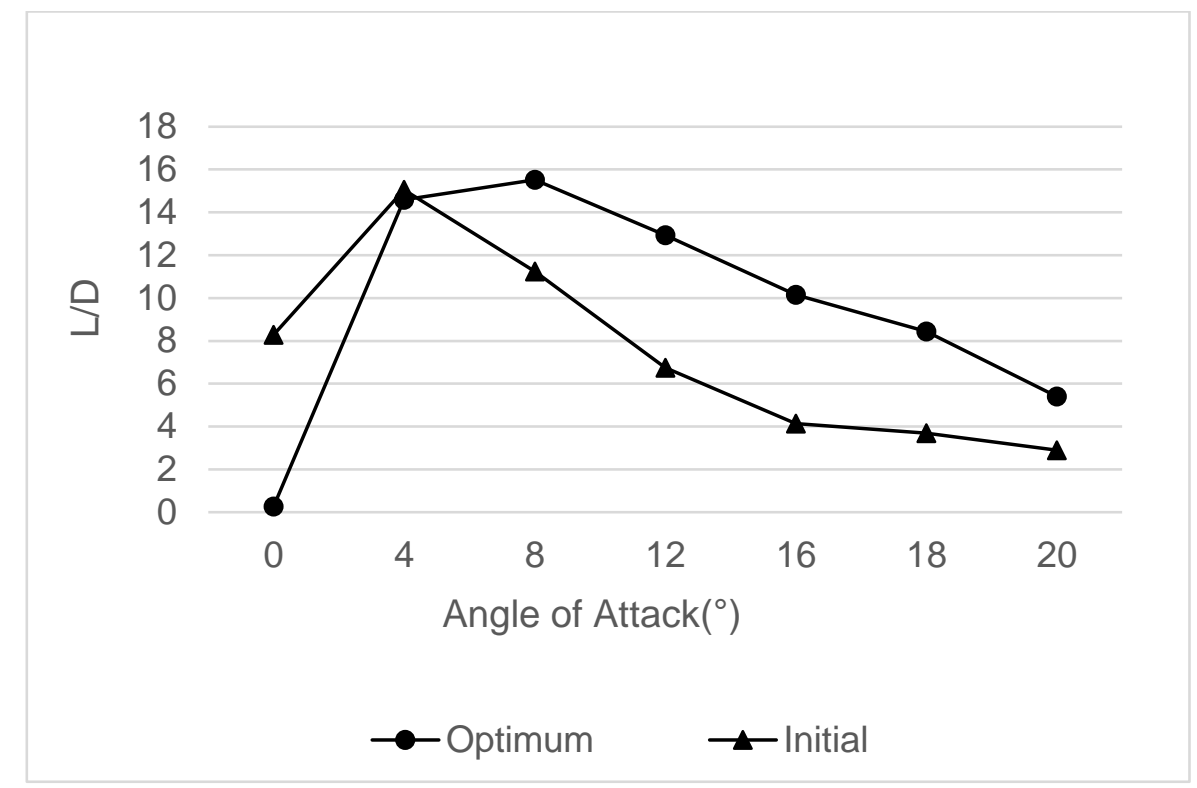

Figure 12. L/D Ratio vs. Angle of Attack, Comparison of Initial and Optimal Designs. 


\section{List of Tables:}

Table 1. Main geometry characteristics of the initial fin.

Table 2. Vector of Design-variables in the Design Space.

Table 3. Design constraints used for RSM.

Table 4. Mesh Sensitivity Study Initial Design

Table 5. Optimal Fin Geometry Characteristics.

Table 6. Results of each iteration 
Table 1. Main geometry characteristics of the initial fin.

\begin{tabular}{lc}
\hline Parameter & Value \\
\hline & \\
Chord Length $(\mathrm{m})$ & 0.1 \\
Depth $(\mathrm{m})$ & 0.12 \\
Sweep Angle $\left({ }^{\circ}\right)$ & 25 \\
Planform & Elliptical \\
Aspect Ratio & 3 \\
Thickness/Chord & 0.09 \\
Section & Half NACA 0009 \\
\hline
\end{tabular}

Table 2. Vector of Design-variables in the Design Space.

\begin{tabular}{lcc}
\hline Parameter & $\begin{array}{c}\text { Lower } \\
\text { Bound }\end{array}$ & $\begin{array}{c}\text { Upper } \\
\text { Bound }\end{array}$ \\
\hline Chord Length (m) & 0.09 & 0.2 \\
Depth (m) & 0.1 & 0.3 \\
Sweep Angle ( ${ }^{\circ}$ ) & 0 & 45 \\
Max Camber (\%) & 0 & 9.5 \\
Max Camber Position (\%) & 10 & 90 \\
Thickness (\%) & 1 & 30 \\
\hline
\end{tabular}


Table 3. Vector of Design-variables in the Design Space.

\begin{tabular}{lcc} 
Parameter & Lower Bound & Upper Bound \\
\hline & & \\
Chord Length (m) & 0.09 & 0.2 \\
Depth (m) & 0.1 & 0.3 \\
Sweep Angle ( $\left.{ }^{\circ}\right)$ & 0 & 45 \\
Max Camber (\%) & 0 & 9.5 \\
Max Camber Position (\%) & 10 & 90 \\
Thickness (\%) & 1 & 30 \\
Drag/Lift & 0 & $\infty$ \\
\hline
\end{tabular}

if Max Camber $=0 \%$ then Max Camber Position=0\%

Table 4. Mesh Sensitivity Study Initial Design

\begin{tabular}{lccc}
\hline & Coarse & Medium & Fine \\
\hline Lift $(N)$ & 49.2 & 50.37 & 50.52 \\
Drag (N) & 6.05 & 6.08 & 6.09 \\
$C_{L}$ & 0.1025 & 0.1049 & 0.1052 \\
$C_{D}$ & 0.0126 & 0.0127 & 0.0127 \\
\hline
\end{tabular}


Table 5. Optimal Fin Geometry Characteristics

\begin{tabular}{lc}
\hline Parameter & Value \\
\hline & \\
Chord Length $(\mathrm{m})$ & 0.182 \\
Depth $(\mathrm{m})$ & 0.274 \\
Sweep Angle $\left({ }^{\circ}\right)$ & 20.288 \\
Max Camber $(\%)$ & 0.059 \\
Max Camber Position $(\%)$ & 85.178 \\
Thickness $(\%)$ & 29.96 \\
Planform Area $\left(\mathrm{m}^{2}\right)$ & 0.0786 \\
Section & Full \\
\hline
\end{tabular}


Table 6. Results of each iteration

\begin{tabular}{|c|c|c|c|c|c|c|c|c|c|}
\hline Parameters & Iter. 1 & Iter. 2 & Iter. 3 & Iter. 4 & Iter. 5 & Iter. 6 & Iter. 7 & Iter. 8 & Iter. 9 \\
\hline Base (mm) & 199.993 & 137.85 & 199.932 & 172.56 & 185.62 & 185.942 & 182.685 & 182.431 & 182.475 \\
\hline Depth (mm) & 279.093 & 232.541 & 241.622 & 299.939 & 250.387 & 242.684 & 273.593 & 278.335 & 274.319 \\
\hline $\begin{array}{l}\text { Sweep Angle } \\
\left(^{\circ}\right)\end{array}$ & 0.02 & 0.018 & 0.003 & 0.119 & 5.695 & 2.062 & 16.135 & 20.421 & 20.288 \\
\hline $\begin{array}{l}\text { Max Camber } \\
(\%)\end{array}$ & 0.027 & 0.044 & 0.062 & 9.449 & 0.101 & 0.048 & 0.005 & 0.032 & 0.059 \\
\hline $\begin{array}{l}\text { Max Camber } \\
\text { Position (\%) }\end{array}$ & 89.97 & 89.99 & 89.967 & 54.61 & 89.973 & 89.87 & 89.151 & 86.255 & 85.178 \\
\hline Thickness (\%) & 29.955 & 29.605 & 29.967 & 20.276 & 29.941 & 29.955 & 29.955 & 29.955 & 29.96 \\
\hline $\begin{array}{l}\text { Drag-per-lift } \\
\text { Ratio (GA) }\end{array}$ & 0.0353 & 0.0574 & 0.0600 & 0.0701 & 0.0652 & 0.0634 & 0.0639 & 0.0643 & 0.0663 \\
\hline $\begin{array}{l}\text { Drag-per-lift } \\
\text { Ratio (CFD) }\end{array}$ & 0.0715 & 0.0742 & 0.0747 & 0.0770 & 0.0723 & 0.0738 & 0.0712 & 0.0706 & 0.0698 \\
\hline
\end{tabular}


2017-05-11

\section{Optimisation of the surfboard fin shape using computational fluid dynamics and genetic algorithms}

Sakellariou, Konstantinos

SAGE

Sakellariou K, Rana ZA, Jenkins KW. Optimisation of the surfboard fin shape using computational fluid dynamics and genetic algorithms. Proceedings of the Institution of Mechanical Engineers, Part P: Journal of Sports Engineering and Technology, 2017, Volume 231, Issue 4, pp344-354

http://dx.doi.org/10.1177/1754337117704538

Downloaded from Cranfield Library Services E-Repository 